\title{
La financiación de RTVE. Qué hay de permanente en un debate que fluctúa ${ }^{1}$
}

\author{
Mercedes MuÑOZ SALDAÑA \\ mms@unav.es \\ Universidad de Navarra
}

Recibido: 5 de marzo de 2014

Aceptado: 27 octubre 2014

\section{Resumen}

El Tribunal de Justicia Europeo archivó en agosto de 2013 el recurso interpuesto por la Comisión europea sobre la compatibilidad del sistema de financiación de RTVE con la normativa comunitaria. El modelo español, aprobado en 2009 y que suprime la publicidad, dispone que los ingresos del ente público se obtienen, entre otros, de: un 3\% de los ingresos de los canales privados de televisión en abierto; un $1.5 \%$ de los ingresos de los canales de pago; y un $0.9 \%$ de los ingresos de los operadores de telecomunicaciones que difunden contenidos audiovisuales. Sin embargo, el debate permanece abierto ya que Redtel (Telefónica, Vodafone, Orange y Ono) interpuso un recurso ante el Tribunal Supremo por un tributo que considera desproporcionado e ilegal. Esta investigación profundiza en el contexto normativo que rodea a la televisión en la actualidad con el objetivo de descubrir qué hay de coyuntural y qué de permanente en la discusión sobre el sostenimiento del servicio público de televisión en España y en Europa. Palabras clave: televisión pública, financiación, servicios de interés económico general, servicio público, publicidad.

\section{RTVE Financiation. What Remains in a Changing Discussion}

\begin{abstract}
The European Court of Justice closed, in August 2013, an action by the European Commission on the compatibility of RTVE's financing with community legislation. The spanish model, approved 2009, which eliminates advertising, envisages that RTVE will obtain 3\% of the income of open private television channels, $1.5 \%$ of that of subscription channels and $0.9 \%$ of that of telecommunications operators that share audiovisual services. However, the national discussion remains open because Redtel, the organization that represents Telefónica, Vodafone, Orange and Ono, appealed to the Supreme Court for a tax as "disproportionate, harmful and illegal". This research analyzes the current legal framework for television in order to discover what's changing and what's stable in the discussion about the funding of public service broadcasting in Spain and Europe.

Keywords: public television, funding, service of general economic interest, public service broadcasting, commercial communication.

\section{Referencia normalizada}

MUÑOZ SALDAÑA, Mercedes (2015): "La financiación de RTVE. Qué hay de permanente en un debate que fluctúa”. Estudios sobre el Mensaje Periodístico. Vol. 21, Núm. 1 (enero-junio), págs.: 455470. Madrid, Servicio de Publicaciones de la Universidad Complutense.

Sumario: 1. Introducción. Qué tipo de servicios son los servicios televisivos. 2. Análisis. Qué es susceptible de ser protegido por su carácter de servicio público. El caso de RTVE. 3. Debate. La controvertida cuestión de la financiación del servicio público televisivo; 3.1. Definición de la misión; 3.2. Adjudicación de la misión; 3.3. Compatibilidad de la prestación con el libre mercado. 4. Conclusiones. Qué hay de permanente en un debate que fluctúa. 5. Referencias: documentos y legislación. 6. Referencias bibliográficas.
\end{abstract}

1 Este artículo está vinculado al Proyecto de Investigación I+D+I (2013-2016): “Transformación de la televisión regional en España: debilitamiento del servicio público y perspectivas de desarrollo tecnológico en un contexto de crisis" (Ref. CS02013-42270-R) 


\section{Introducción. Qué tipo de servicios son los servicios televisivos}

El Presidente de la Corporación RTVE calificaba en 2012 la situación de la televisión pública española de insostenible y cuestionaba, al margen de su legalidad, el sistema de financiación establecido por el Gobierno español tres años antes. Por su parte, el Ministro de Hacienda anunciaba en ese mismo año que habría modificaciones en dicho modelo. Sin embargo, el Presidente actual de la Corporación apuesta por mantener el sistema vigente.

Resulta inevitable advertir que en el debate se emplean simultáneamente los mismos argumentos para defender posturas contrapuestas: preservar el servicio público de radiodifusión; velar por su independencia ante los intereses comerciales; apostar por la calidad; garantizar la competencia leal; defender los derechos de los consumidores; o salvaguardar el pluralismo son sólo algunos de los argumentos que, desde las tribunas jurídicas, políticas y profesionales aparecen para defender esquemas opuestos (Arriaza, 2012).

En cualquier caso, a pesar del archivo del caso por la parte de la Comisión, la discusión permanece abierta ya que, RTVE, con una situación económica en la cuerda floja, cerró el ejercicio de 2013 con un déficit superior a los 70 millones de euros, una cifra elevada, aunque inferior a los 113 millones de euros de pérdidas de $2012^{2}$. La polémica sobre la posible vuelta de la comunicación comercial a RTVE sigue latente, decisión que, sin duda, desencadenaría que las televisiones privadas, grandes defensoras del sistema actual, desenterraran el hacha de guerra. El problema que rodea a la definición y financiación del servicio público de televisión, aunque histórico, no es incomprensible. Sin embargo, para profundizar en él, hay que enmarcarlo en un debate más amplio que afecta a la ordenación del mercado audiovisual europeo en su conjunto y que parte de la compleja naturaleza de los servicios televisivos (Muñoz Saldaña, 2011).

La Comisión Europea ratificaba en 2009, en la carta enviada a las instituciones españolas y en la cual cuestionada inicialmente el sistema de financiación adoptado en España para la televisión pública, que "RTVE es una empresa a la que se le ha encomendado la prestación de un servicio de interés económico general (en adelante, SIEG)". Este concepto es propio del régimen jurídico comunitario y, hasta la aprobación de la Ley 7/2010, de 31 de marzo, General de Comunicación Audiovisual, resultaba ajeno a legislación audiovisual española. El artículo 43.2 de esta ley dispone, en referencia a la prestación del servicio público de comunicación audiovisual, que: "los servicios de interés económico general de comunicación audiovisual radiofónica, televisiva, conexos e interactivos de titularidad estatal no admitirán ninguna forma de comunicación comercial audiovisual, ni la emisión de contenidos audiovisual en sistemas de acceso condicional, sin perjuicio de las excepciones que su normativa específica de financiación establezca".

¿Qué supone la consideración de los servicios televisivos como SIEG? Siguiendo la jerarquía de las fuentes comunitarias, conviene partir del papel que los Tratados de la Unión otorgan a este tipo de servicios (Gómez Barroso y Marban-Flores, 2013). Fiel

2 Disponible en: http://ecoteuve.eleconomista.es/ecoteuve/empresas/noticias/5145065/09/13 /RTVE-preve-terminar-el-ejercicio-2013-con-un-deficit-de-al-menos-70-millones.html\#.Kku8LWZjmG5215f [Consultado el 26 de febrero de 2014]. 
a sus principios económicos liberalizadores, el Derecho comunitario no regula de forma explícita la técnica del servicio público como configuración jurídica, que es la que tradicionalmente se aplicaba a la televisión pública en España y en la mayoría de países de la Unión Europea. Así, aunque el Tratado de Funcionamiento de la Unión Europea (en adelante, TFUE) incluye entre sus disposiciones la referencia a determinadas "obligaciones inherentes a la noción de servicio público" (art. 93), a efectos prácticos, la concibe como una situación excepcional cuya protección jurídica y régimen extraordinario se integra en el régimen previsto para las actividades calificadas como SIEG.

De acuerdo este planteamiento, el art. 106.2 TFUE dispone que "las empresas encargadas de la gestión de servicios de interés económico general o que tengan en carácter de monopolio fiscal, quedarán sometidas a las normas del presente Tratado, en especial, a las normas sobre la competencia, en la medida en que la aplicación de dichas normas no impida de hecho o de derecho, el cumplimiento de la misión a ellas confiada [...]". Es decir, este artículo parte del normal sometimiento de todas las empresas encargadas de prestar un SIEG a las leyes del mercado. Sin embargo, al mismo tiempo que instaura esta regla general, les atribuye misiones específicas y, por ello, contempla una posible excepcionalidad en el caso de que dichas misiones no puedan ser satisfechas en un ámbito de libre competencia.

En relación con la posibilidad de aplicar un régimen excepcional a las empresas que prestan un SIEG, el art. 14 TFUE reconoce que "a la vista del lugar que los servicios de interés económico general ocupan entre los valores comunes de la Unión [...] los Estados miembros, con arreglo a sus competencias respectivas y en el ámbito de aplicación de los Tratados, velarán por que dichos servicios actúen con arreglo a principios y condiciones, en particular económicas y financieras, que les permitan cumplir su cometido [...]". Este artículo fue introducido por el Tratado de Ámsterdam con el objetivo de equilibrar la aplicación comunitaria de las leyes del mercado y la intención por parte de los Estados de garantizar la continuidad de determinados servicios públicos nacionales.

Además de estos dos artículos, resulta destacable la incorporación al acervo comunitario del Protocolo $\mathrm{n}^{\mathrm{o}} 26$, anexo al TFUE, el cual reconoce que: "Los valores comunes de la Unión con respecto a los servicios de interés económico general [...] incluyen en particular:

a) El papel esencial y la amplia capacidad de discreción de las autoridades nacionales, regionales y locales para prestar, encargar y organizar los servicios de interés económico general lo más cercanos posible a las necesidades de los usuarios;

b) La diversidad de los servicios de interés económico general y la disparidad de las necesidades y preferencias de los usuarios que pueden resultar de las diferentes situaciones geográficas, sociales y culturales;

c) Un alto nivel de calidad, seguridad y accesibilidad económica, la igualdad de trato y la promoción del acceso universal y de los derechos de los usuarios".

En esta misma línea, la Carta Europea de Derechos Fundamentales en su artículo 36 "reconoce y respeta el acceso a los servicios de interés económico general, tal como disponen las legislaciones y prácticas nacionales, de conformidad con el Tratado cons- 
titutivo de la Comunidad Europea, con el fin de promover la cohesión social y territorial de la Unión".

En resumen, el Derecho primario asume que los servicios calificados como SIEG, por la naturaleza de las prestaciones que satisfacen: ocupan un lugar destacado tanto en el contexto nacional como en el comunitario; conllevan el cumplimiento de una serie de misiones de servicio público; deben someterse, como norma general, a las leyes del mercado; y pueden ser objeto de un régimen excepcional debidamente justificado y orientado a garantizar cumplimiento de las misiones especiales que se les atribuye. El TFUE establece éstas como las bases de la configuración jurídica de los SIEG, aunque han sido las instituciones europeas, tanto la Comisión a través de diferentes textos como las decisiones del Tribunal de Justicia Europeo, las que han facilitado el desarrollo de dicha categoría y la aplicación de los principios generales que rigen el funcionamiento de estos servicios a actividades concretas.

Desde mediados de los noventa la construcción de un marco jurídico estable para la categoría de los SIEG se ha constituido como uno de los retos presentes en la política europea en materia de libre prestación de servicios. Esta tarea tiene sus primeros antecedentes representativos en 1996 con la Comunicación de la Comisión "Los servicios de interés general en Europa" (COM (1996) 443 final), a la cual han sucedido decenas de documentos.

Entre los más recientes, la Comisión aprobó en 2012 un paquete de medidas con el objetivo de clarificar el régimen jurídico de los SIEG y, más en concreto, de las ayudas que los Estados pueden otorgarles en compensación por prestación de servicios públicos a la sociedad. Estos textos son: la "Decisión de la Comisión relativa a la aplicación de las disposiciones del artículo 106, apartado 2, del Tratado de Funcionamiento de la Unión Europea a las ayudas estatales en forma de compensación por servicio público concedidas a algunas empresas encargadas de la gestión de servicios de interés económico general" (Do L 7 de 11-1-2012. pp.3-10); la "Comunicación de la Comisión. Marco de la Unión Europea sobre ayudas estatales en forma de compensación por servicio público" (DO C 8, de 11.1.2012, pp. 15-22); la "Comunicación de la Comisión relativa a la aplicación de las normas de la Unión Europea en materia de ayudas estatales a las compensaciones concedidas por la prestación de servicios de interés económico general" (DO C 8. de 11.1.2012, pp. 4-14); y el Reglamento $\mathrm{n}^{\circ} 360$ relativo a la aplicación de los artículo 107 y 108 del Tratado de Funcionamiento de la Unión Europea a las ayudas de minimis concedidas a la empresas que prestan servicios de interés económico general, (DO L 114, de 26.4.2012, pp. 8- 13).

El marco normativo de la financiación de la televisión pública en Europa debe interpretarse teniendo en cuenta dichos documentos con el eje central de la "Comunicación de la Comisión sobre la aplicación de las normas en materia de ayudas estatales a los servicios públicos de radiodifusión" adoptada en 2001 y actualizada en 2009 (DO C 257, de 27.10.2009, pp. 1-14).

Además de lo señalado en los Tratados, los textos europeos han incidido en la necesidad inicial de definir qué es un SIEG, aceptando, de una forma amplia, que son: "actividades de servicio comercial que cumplen misiones de interés general y están por ello sometidas, por parte de los Estados miembros, a obligaciones específicas de ser- 
vicio público" (COM (2003) 270 final, punto 17, p. 7). A este respecto, "los servicios de interés económico general se diferencian de los servicios ordinarios en que los poderes públicos consideran que deben prestarse incluso cuando el mercado puede no tener suficientes incentivos para hacerlo" (COM (2000) 580 final, punto 14). Así, la Comisión Europea ha señalado que "no sería adecuado vincular obligaciones específicas de servicio público a una actividad que ya efectúan, o pueden efectuar satisfactoriamente [...] empresas que operan en condiciones normales de mercado" (2012/C $8 / 02$, punto 48 ). De esta forma, dimensión comercial y presencia de un interés público son los ejes que permiten identificar a un SIEG.

Además, en 2012 se ha destacado que "el concepto de servicio de interés económico general es un concepto cambiante que depende, entre otras cosas, de las necesidades de los ciudadanos, la evolución tecnológica del mercado" e, incluso, de "las preferencias sociales y políticas en el Estado Miembro en cuestión" (2012/C 8/02, punto 45).

Añadido a estas consideraciones de carácter general, la Comisión Europea ha proporcionado una serie de criterios que permiten identificar el interés público que distingue a los SIEG de los servicios ordinarios y que se concreta en: la necesidad de prestar un servicio universal; la obligación de garantizar la continuidad de la actividad; la necesidad de preservar la asequibilidad del servicio; y la especial protección de los derechos de los consumidores y de los usuarios respecto de dicho servicio. Estos criterios identificativos, específicos de los SIEG, pueden satisfacerse bien en un régimen de libre mercado o bien mediante un régimen excepcional debidamente justificado de acuerdo con lo establecido por el art. 106.2 y 14 del TFUE.

A pesar de que el marco jurídico que afecta a estas actividades es de origen y desarrollo comunitarios y de que la Comisión asume una competencia supervisora sobre la adecuación del funcionamiento a las reglas del Mercado Único, los Estados Miembros, en teoría, son los principales responsables en la tarea de identificación, organización y control de las actividades calificadas como SIEG.

Este planteamiento está presente en el Protocolo $n^{\circ} 26$ cuando se reconoce el papel esencial y la amplia discrecionalidad de las autoridades, no sólo nacionales, sino también regionales y locales, en lo referente a la organización y prestación de los SIEG. El protagonismo de las autoridades nacionales resulta lógico ya que las necesidades sociales de los Estados miembros, aunque con puntos de encuentro, varían dependiendo del contexto social, político, económico o cultural y, por ello, como sostiene la Comisión: "los Estados miembros disponen de un amplio margen de apreciación a la hora de definir un determinado servicio como SIEG y de conceder una compensación al proveedor del servicio" ((2012/C 8/02, punto 46).

Las competencias de la Comisión al respecto se limitan a comprobar si el Estado miembro ha incurrido en un error manifiesto al definir el servicio como SIEG y al evaluar la ayuda estatal contenida en la compensación. Sin embargo, la historia demuestra que la identificación y definición de actividades como SIEG ha resultado una labor conjunta de las instituciones nacionales y comunitarias, con especial protagonismo de estas últimas. Este ha sido el caso de la televisión.

Ya en el año 2000 la Comisión declaraba que, a pesar de las reservas nacionales respecto de estos mercados, "hoy en día los sectores de televisión y la radio están libe- 
ralizados a escala comunitaria" aunque reconocía la potestad de los Estados miembros para decidir, bajo supervisión comunitaria, "si quieren establecer un sistema de radiodifusión de servicio público, definir su mandato exacto y establecer las modalidades de su financiación" (COM (2000) 580 final, p. 39). Y en esas estamos. Las autoridades españolas, dentro del régimen dispuesto para los SIEG son las responsables de definir en qué consiste el mandato de servicio público que atribuyen a RTVE y de justificar la excepcionalidad y cuantía de su financiación. Por su parte, las autoridades comunitarias asumen el papel de garantizar que dicha financiación se ajusta a lo dispuesto en el TFUE.

\section{Análisis. Qué es susceptible de ser protegido por su carácter de servicio pú- blico. EI caso de RTVE}

Siguiendo lo establecido en el art. 106.2 del TFUE, las empresas encargadas de prestar un SIEG son empresas a las cuales se les ha encomendado una misión específica, una misión de servicio público. Se considera que dicha empresa no asumiría dicha misión si tuviera exclusivamente en cuenta su interés comercial, o al menos no la asumiría en la misma medida ni en las mismas condiciones.

Aunque, como se ha señalado, los Estados miembros tiene un papel primordial en la identificación y definición de misiones de servicio público, las instituciones comunitarias han apuntado como obligaciones de servicio público comunes a los SIEG las siguientes:

- La prestación de un servicio universal: se considera que "este concepto establece el derecho de toda persona a acceder a determinados servicios considerados esenciales e impone a los proveedores la obligación de prestar servicios concretos en condiciones precisas, con una cobertura territorial completa y a un precio asequible" (COM (2004) 374 final, p. 8). Las instituciones comunitarias han incidido tanto en la flexibilidad como en el carácter evolutivo de un término que debe adaptarse a la circunstancias del sector al cual se aplique.

- La calidad del servicio: la necesidad de alcanzar unos niveles de calidad socialmente aceptables puede justificar por sí sola la imposición de una serie de obligaciones de servicio público a través de una regulación específica. A pesar de que no existe consenso acerca de una definición general de calidad, ésta se asocia a cuestiones como una adecuada cobertura territorial, la continuidad de la prestación, la transparencia financiera, la asequibilidad del servicio y la salvaguarda de los derechos de consumidores o usuarios.

- Protección de consumidores y usuarios, en especial de colectivos como menores.

Las autoridades nacionales, teniendo en cuenta estos criterios generales, tienen un papel protagonista en la concreción definitiva de las misiones de servicio público que corresponden a cada sector y, de acuerdo con esta competencia, pueden tomar decisiones en el ámbito nacional, regional o local, siempre que tengan en cuenta el concepto comunitario de los SIEG.

Por ello, la Comisión, al cuestionar el régimen audiovisual español, analizaba la definición de la misión de servicio público aprobada en la Ley 17/2006, de 5 junio, de la radio y la televisión de titularidad estatal, modificada por la Ley 8/2009, de 28 de 
agosto de financiación de la Corporación de Radio y Televisión Española y completada por la Ley 7/2010, de 31 de marzo, General de Comunicación Audiovisual.

En el caso de la televisión, la Ley 17/2006, de 5 junio, de la radio y la televisión de titularidad estatal atribuye la calificación de servicio público a las actividades encomendadas a la corporación RTVE y, así, en su artículo 2 califica este servicio como: "esencial para la comunidad y la cohesión de las sociedades democráticas"; cuya función abarca:

- "la producción de contenidos y la edición y difusión de canales generalistas y temáticos, en abierto o codificados, en el ámbito nacional e internacional, así como la oferta de servicios conexos o interactivos";

- con la finalidad de "satisfacer necesidades de información, cultura, educación y entretenimiento de la sociedad española";

- de "difundir su identidad y diversidad culturales";

- de "impulsar la sociedad de la información";

- y de "promover el pluralismo, la participación y los demás valores constitucionales, garantizando el acceso de los grupos sociales y políticos significativos a través de la prestación de un servicio de cobertura universal, entendiendo por tal la mayor cobertura posible del territorio nacional".

Esta definición, acorde con los parámetros generales de servicio público atribuidos de forma general a los SIEG se completa en el art. 3 de esta misma Ley nacional con un amplio listado de funciones específicas que competen a RTVE. Entre estas funciones se incluyen:

- garantizar una información objetiva, veraz y plural;

- facilitar el debate y la libre expresión de opiniones;

- promover el derecho de acceso a los medios;

- ofrecer acceso a los distintos géneros de programación y a los acontecimientos institucionales, sociales, culturales y deportivos de interés general;

- apoyar la integración de las minorías;

- asegurar la máxima continuidad y cobertura geográfica y social con el compromiso de ofrecer calidad, diversidad, innovación y exigencia ética;

- preservar los derechos de los menores.

Por su parte, la Ley 8/2009, de 28 de agosto, de financiación de la Corporación de Radio y Televisión Española añade, en su artículo 9 (capítulo V), sin perjuicio de las anteriores, otro extenso listado de "obligaciones adicionales de servicio público", que también competen a RTVE, y que incluye, entre otras, las siguientes misiones:

- incorporar en sus programas "servicios interactivos en los que se de acceso a los grupos políticos, sindicales y sociales";

- informar sobre los debates parlamentarios y emitir en directo "las sesiones que tengan un especial interés público";

- emitir programas y debates electorales;

- emitir en el canal infantil, los días laborales entre las diecisiete y las veintiún horas locales y los fines de semana y festivos entre las nueve y las veinte horas locales, al menos un 30\% de programas destinados a niños de entre 4 y 12 años. 
La Ley 7/2010, de 31 de marzo, General de Comunicación Audiovisual tampoco renuncia a contribuir en la definición el servicio público de comunicación audiovisual y bajo la consideración de que "el servicio público de comunicación audiovisual es un servicio esencial de interés económico general" dispone en su artículo 40 que dichos servicios tiene como misión:

- difundir contenidos que fomenten los principios y valores constitucionales;

- contribuir a la formación de una opinión pública plural;

- dar a conocer la diversidad cultural y lingüística de España;

- difundir el conocimiento y las artes, con especial incidencia en el fomento de una cultura audiovisual;

- Atender a aquellos ciudadanos y grupos sociales que no son destinatarios de la programación mayoritaria.

Además, la Ley General Audiovisual, condiciona el cumplimiento de dicha misión a la producción, edición y difusión de un conjunto de canales de radio, televisión y servicios de información en línea con programaciones diversas y equilibradas para todo tipo de público, cubriendo todos los géneros, destinadas a satisfacer las necesidades de información, cultura, educación y entretenimiento de la sociedad y a preservar el pluralismo en los medios de comunicación" (art. 41).

Esta sucesión de obligaciones generales de servicio público y la modificación o permanente ampliación del listado original a tan sólo dos años de su aprobación evidencian la dificultad o quizá se podría hablar de la imposibilidad de definir con precisión y estabilidad una serie de misiones que sean competencia exclusiva de los entes públicos o de distinguir entre las prestaciones ofrecidas por las cadenas que asumen estas obligaciones y el resto de las cadenas comerciales. Los primeros asuntos que llegaron al Tribunal de Justicia Europeo relativos a este asunto ya evidenciaban dicha dificultad. Así, la Comisión Europea justificaba las medidas excepcionales aplicadas a las televisiones públicas en "la obligación de ofrecer una programación variada que comprenda programas culturales, educativos, científicos destinados a minorías y [...] la de alcanzar a toda la población nacional, independientemente del coste" (Decisión 93/403/CEE, DO L 179, p. 23).

De hecho, en 2009, la Comisión Europea reconocía que, dada la naturaleza específica de radiodifusión, cabe considerar legítima una definición de misión de servicio público de carácter cualitativo "que encomiende a un operador determinado la obligación de suministrar una amplia gama de programas y una oferta de programación equilibrada y variada". Incluso, dicha definición puede reflejar el desarrollo y la diversificación de las actividades en la era digital e incluir servicios audiovisuales en todas las plataformas de distribución" (2009/C 257/01, punto. 47).

Por razones diversas (de carácter histórico, económico, tecnológico e incluso político) las instituciones comunitarias han asumido, no sin dificultad y oposición, la legitimidad de la posición privilegiada concedida a las cadenas públicas en el mercado europeo como garantes de la satisfacción de estas misiones de servicio público. Sin embargo ese privilegio, aunque asumido o tolerado, no es ilimitado. 


\section{Debate. La controvertida cuestión de la financiación del servicio público tele- visivo}

La Comunicación de la Comisión sobre la aplicación de las normas en materia de ayudas estatales a la financiación de los servicios públicos de radiodifusión, publicada en 2001 (2001/C 320/04) y actualizada en 2009 (2009/C 257/01) es un documento de referencia en el estudio sobre la polémica financiación de RTVE. La carta remitida por la Comisión a las autoridades españolas hace referencia explícita a este texto como marco en el que se integra la evaluación de lo ocurrido en el caso español. Este documento tiene el objetivo de establecer los criterios que debe seguir la Comisión a la hora de aplicar el régimen expuesto (el aplicable a los SIEG, en concreto los artículos 107 y 106.2 del TFUE) a los servicios públicos de radiodifusión.

La Comisión, al afrontar el espinoso asunto de la financiación del servicio público de radiodifusión, asume como punto de partida que este tipo de servicios, "a pesar de tener una importancia económica evidente, no son comparables con los servicios públicos de cualquier otro sector económico" ya que "no existe ningún otro servicio que, simultáneamente, tenga un acceso tan amplio a la población, le proporcione tal cantidad de informaciones y contenidos y, de este modo, transmita a las opiniones individuales y la opinión pública e influya tanto en las mismas", además, "de modo generalizado, la radiodifusión se considera una fuente de información fiable, e incluso la principal para una parte no menos preciable de la población" que, en último término "garantiza a todos los ciudadanos un grado equitativo de participación en la vida pública" (2001/C 320/04, puntos 6 y 7; 2009/C 257/01, puntos 9 y 10). Por estos motivos, se entiende que las televisiones públicas, como depositarias de las obligaciones de servicio público, detentan la responsabilidad de satisfacer las necesidades y las obligaciones de interés público que, de lo contrario, no necesariamente resultarían satisfechas en grado óptimo.

El cumplimiento de dichas misiones exige garantizar la viabilidad económica de su prestación y, por ello, el Derecho comentario prevé la posibilidad de financiarlo de manera excepcional. Para que dicha financiación extraordinaria, prevista en el art. 106.2 TFUE, no sea considerada una ayuda incompatible con las normas del Tratado debe cumplir, básicamente, tres requisitos:

a) El servicio en cuestión debe ser un SIEG y estar definido claramente como tal por el Estado miembro: definición.

b) La empresa en cuestión debe haber sido encargada explícitamente por el Estado miembro del suministro de dicho servicio: misión.

c) La aplicación de las normas de competencia del Tratado (en este caso concreto, la prohibición de ayudas estatales) debe obstaculizar el cumplimiento de las tareas específicas asignadas a la empresa y la excepción a dichas normas no debe afectar al desarrollo de intercambios en una medida contraria al interés de la Comunidad: proporcionalidad.

Corresponde a la Comisión Europea verificar el cumplimiento de los criterios anteriores. 


\subsection{Definición de la misión}

El primer requisito para justificar un régimen económico excepcional reside en la definición de la misión a garantizar. Como se ha desarrollado en el apartado anterior, esta definición es competencia principal de los Estados Miembros atendiendo al concepto general de los SIEG y a los criterios desarrollados por las instituciones comunitarias. Aquí, teniendo en cuenta lo que se ha señalado con anterioridad, nos encontramos los primeros problemas al aplicarlo a las actividades televisivas. Definir implica acotar, delimitar el significado de algo de tal forma que permita distinguir ese algo del resto.

Sin embargo, la definición de servicio público televisivo contenida en la legislación audiovisual española es tan amplia y general que resulta imposible no atribuir muchas de sus características a las actividades prestadas por las televisiones privadas a las que, en principio, no se les atribuye la definición de misión de servicio público ni se acogen a medidas excepcionales. La obligación de satisfacer necesidades de información, de cultura o de entretenimiento; la misión de difundir contenidos que fomenten valores y principios constitucionales; o la de preservar los derechos de los menores resultan exigencias comunes a la prestación de servicios televisivos, sin embargo, integran la definición expresa de servicio público de radiodifusión.

Esta problemática también está presente en algunos de los planteamientos del texto europeo, el cual determina, por un lado que, "la definición de misión de servicio público debe ser tan concreta como sea posible" o que "no debe dejar ninguna duda sobre si el Estado Miembro pretende que se incluya o no en la misión de servicio público determinada actividad realizada por el operador al que se encomienda dicha misión" o que "es importante determinar claramente las actividades incluidas en la misión de servicio público [...] a fin de que las autoridades [...] puedan controlar eficazmente su cumplimiento" (2009/C 257/01, puntos 45 y 46).

Sin embargo, al mismo tiempo, reconoce que cabe considerar legítima "una definición cualitativa que encomiende a un operador determinado la obligación de suministrar una amplia gama de programas y una oferta de programación equilibrada y variada" (2009/C 257/01, punto 47.); que dicha definición también puede reflejar el desarrollo y la diversificación de las actividades en la era digital e incluir servicios audiovisuales en todas las plataformas de distribución" (2009/C 257/01, punto 47.); o asumir que los operadores comerciales también contribuyen a satisfacer las misiones de servicio público "en la medida en que contribuyen a garantizar el pluralismo, enriquecen el debate cultural y político y amplían la oferta de programas" (2009/C 257/01, punto16).

La Comisión asume esta problemática y entiende que, en el caso de la televisión, aunque la definición de servicio público debe hacerse teniendo en cuenta el concepto comunitario de los SIEG, "cabe considerar legítima [...] una definición amplia que confíe a un operador determinado la tarea de ofrecer una programación equilibrada y variada, acorde con su función, manteniendo al mismo tiempo cierto nivel de audiencia" (2001/C 320/04, punto 33).

Así la institución comunitaria con esta definición amplia y general de servicio público televisivo legitima expresamente la existencia de las televisiones públicas europeas y su papel garante en la satisfacción de las misiones de servicio público. Sin embargo dicha definición también tiene límites. En lo referente a dichos límites, la 
labor de la Comisión se limita a controlar los errores manifiestos, tales como incluir en la calificación de servicio público actividades de las que no pueda razonablemente derivarse la satisfacción de necesidades democráticas, sociales o culturales de cada sociedad como: el comercio electrónico, la publicidad comercial, el uso de números de teléfono especiales en juegos con premio, o la televenta.

Este planteamiento se reafirma en la jurisprudencia comunitaria, la cual ha corroborado con sus fallos la posibilidad de incluir una programación generalista de calidad dentro del concepto servicio público de radiodifusión y, más en concreto, aplicada a la obligación de garantizar la prestación de un servicio universal televisivo de calidad tanto desde el punto de vista técnico como en lo referente a la programación.

\subsection{Adjudicación de la misión}

En un segundo paso, el Derecho comunitario exige, para la justificación de la financiación adicional de los servicios públicos de radiodifusión, que dichas misiones hayan sido atribuidas expresamente a un operador en concreto en virtud de un instrumento oficial (por ejemplo, legislación, contrato o pliego de condiciones). En el caso de España dicha atribución se realiza a través de la Ley 17/2006, de 5 de junio, de radio y televisión de titularidad estatal mediante la cual 1. Se atribuye a la Corporación de Radio y Televisión Española, S. A., Corporación RTVE, la gestión del servicio público de radio y televisión en los términos que se definen en esta Ley, para ser ejercido directamente por las sociedades filiales de la Corporación prestadoras de los servicios de radio y televisión. Esta atribución se concreta a través de los Mandatos-marco mediante los cuales las Cortes Generales concretan los objetivos de la función de servicio público que se encomienda a RTVE por períodos de 9 años. Dichos objetivos son desarrollados cada 3 años a través de los contratos-programas acordados por el Gobierno con la Corporación RTVE.

La Comunicación de la Comisión al afrontar este requisito da por hecho, quizá erróneamente, que dicha misión es competencia necesaria de los entes públicos y por ello sostiene que no basta con que al organismo público se le haya encargado formalmente dicha prestación, sino que dicho servicio debe prestarse de manera efectiva en las condiciones pactadas y, para ello, es necesario que una autoridad competente $\mathrm{u}$ organismo al efecto controle su aplicación de manera transparente y eficaz. La elección del mecanismo destinado a controlar dicho cumplimiento es una competencia exclusiva de los Estados miembros (2001/C 320/04, puntos 41 y 42; 2009/C 257/01, punto 54). La Comisión le otorga tal importancia a la supervisión que especifica que, a falta de información clara y fiable sobre el cumplimiento de la misión de servicio público, la Comisión no podría justificar la adopción de ninguna medida excepcional aplicable a su satisfacción.

\subsection{Compatibilidad de la satisfacción de la misión con el libre mercado}

El tercer requisito se refiere a las condiciones que se imponen a la financiación de los servicios público de radiodifusión de tal forma que dicha financiación no resulte contraria a las leyes de la competencia. En este apartado, se aplican los principios de necesidad y de proporcionalidad para estudiar la compatibilidad con las leyes del mercado de la concesión de ayudas para la satisfacción de misiones de servicio público. 
Desde una perspectiva general los sistemas de financiación televisiva pueden dividirse en dos grandes grupos: de financiación única o de doble financiación. La doble financiación (proveniente de fondos públicos y publicidad $u$ otros ingresos comerciales) ha desencadenado numerosas acusaciones por competencia desleal por parte de los entes comerciales que en muchos casos han llegado a instancias europeas hasta convertir la financiación de los servicios públicos televisivos en el asunto más polémico del presente y futuro del mercado audiovisual en Europa. Y es que, en lo referente a la financiación, en principio, los Estados miembros son los responsables de optar por un régimen u otro y la Comisión vigila (según lo dispuesto en el art. 106.2 TFUE) que el régimen elegido por las autoridades nacionales resulte compatible con la normativa europea.

En relación a este último requisito, el Derecho comunitario no cuestiona, de por sí, ni el sistema de financiación estatal ni el sistema de financiación mixta. Sobre la primera posibilidad, tal y como recoge el Protocolo sobre Radiodifusión del Tratado de Ámsterdam "las disposiciones del Tratado constitutivo de la Comunidad Europea se entenderán sin perjuicio de la Facultad de los Estados miembros de financiar el servicio público de radiodifusión en la medida en que la financiación se conceda a los organismos de radiodifusión para llevar a cabo la función de servicio público tal y como haya sido atribuida, definida y organizada por cada Estado miembro, y en la medida en que dicha financiación no afecte a las condiciones del comercio y de la competencia en la Comunidad en un grado que sea contrario al interés común, debiendo tenerse en cuenta la realización de la función de dicho servicio público".

Entre las ventajas derivadas de este sistema se ha señalado que "permite repartir la carga que supone la financiación de una misión de servicio público entre todos los contribuyentes. Este modo de financiación no frena el acceso al mercado y está sujeto al control parlamentario en el marco del procedimiento presupuestario de los Estados Miembros", COM (2003) 250 final, punto 62, apartado a), p. 58.

$\mathrm{Y}$, en relación a la segunda, "no puede plantearse en principio ninguna objeción a la elección de un sistema de doble financiación, que combine fondos públicos e ingresos por publicidad, en vez de un solo sistema de financiación, solamente fondos públicos, mientras no se afecte a la competencia en los mercados competentes, a saber, publicidad y adquisición o venta de programas) en una medida contraria al interés comunitario" (COM (2000) 580 final, punto 5, p. 38). La Comisión, en el texto de 2009, fija su atención en explicar cuáles son los requisitos para que la financiación mixta de las televisiones públicas, como principales responsables de la satisfacción de las misiones de servicio público, resulte compatible con el Derecho comunitario. Estos requisitos se pueden resumir en: necesidad, transparencia, control y proporcionalidad de las ayudas concedidas.

Sin embargo, la aplicación de dichos principios no resulta sencilla en un ámbito en el que: se contempla una definición de misión de servicio público amplia y general, de carácter cualitativo; los operadores comerciales comparten con los entes públicos de manera inevitable ciertas obligaciones inherentes a su actividad profesional; y, por tanto, no resulta fácil la separación de cuentas ente las actividades de servicio público de las que no lo son. 
Por ello, la Comisión Europea considera que la transparencia financiera puede incrementarse más "mediante la separación adecuada entre las actividades de servicio público y las que no lo son a nivel de la organización del organismo público de televisión" (2009/C 257/10, punto 69).

En cualquier caso, la financiación es sin duda el asunto más espinoso de la agenda. De hecho, en la última década, la Comisión ha estudiado, entre otros casos, la legalidad de los sistemas de financiación de: Italia, Portugal, Dinamarca, Francia, Holanda, Alemania y España ${ }^{3}$. En las demandas de los operadores privados ante la Comisión Europea destaca en la actualidad su oposición a la diversificación, financiada con fondos públicos, de las actividades de los operadores estatales en sectores como los contenidos en línea o los canales especializados.

Aunque una valoración rigurosa de estos principios organizaciones precisa de un análisis más detenido, estos tres requisitos y su materialización en la legislación audiovisual española obligan a considerar, en primer lugar, que quizá convendría asumir definitivamente la imposibilidad de concretar jurídicamente, con precisión y de tal forma que se distinga del resto de prestaciones (más allá de lo que supone la prestación de un servicio universal televisivo), en qué debe consistir el servicio público de televisión y reconocer que su definición se deriva de que constituye un requisito imprescindible para su configuración jurídica directamente vinculada con el segundo requisito: su atribución a un ente específico. De hecho, la propia Dirección General de Competencia de la Comisión reconoce expresamente que la obligación de definir el servicio público se vincula al objetivo de minimizar las repercusiones negativas que la financiación estatal de estas prestaciones puede ocasionar en el funcionamiento del libre mercado televisivo ${ }^{4}$. Por otro lado, también conviene asumir que dicha atribución, en el caso español a RTVE, resulta tan sólo una opción más de entre las posibles ya que la naturaleza del servicio que presta el ente público es compartida con la actividad desarrollada por los entes comerciales.

Una cuestión obvia, aunque olvidada desde instancias políticas y profesionales, es que lo que las instituciones comunitarias han definido y desarrollado en sus documentos como servicio público de radiodifusión no es de por sí equivalente al concepto televisión pública. Por eso se puede sostener que el modelo de funcionamiento de los operadores públicos es una cuestión discutible en la que elementos como el desarrollo y crecimiento del mercado, las posibilidades tecnológicas o la coyuntura política pueden jugar un papel fundamental. Sin embargo, el futuro de lo que se califica como el servicio público de radiodifusión genera un debate mucho más de fondo en el que no se cuestiona la continuidad de unas obligaciones de servicio público cuyo cumplimiento debe ser supervisado por las autoridades correspondientes, a poder ser independientes del poder político y empresarial, y que genera unos derechos ciudadanos de obligada garantía independientemente de las circunstancias del mercado, de la coyuntura política y del mayor o menor grado de desarrollo tecnológico de aquí en adelante.

3 "Legal Framework conditions applicable to State aid to public service broadcasting" en: http://ec.europa.eu/competition/sectors/media/decisions_psb.pdf [Consultado el 3 de marzo de 2014].

4 "Competition in the Media Sector" en: http://ec.europa.eu/competition/sectors/media/overview_en.html. [Consultado el 30 de septiembre de 2013]. 


\section{Conclusiones. Qué hay de permanente un debate que fluctúa}

Una vez analizado el marco de la categoría jurídica de la SIEG y su repercusión en los servicios televisivos, se pueden sostener, entre otras las siguientes conclusiones:

- La calificación de los servicios televisivos como SIEG resulta adecuada a la compleja naturaleza de esta actividad ya que el Derecho comunitario considera asumible dentro de esta categoría que los Estados protejan la prestación y satisfacción de determinadas misiones de servicio público, adoptando medidas excepcionales debidamente justificadas.

- El interés público que distingue este tipo de actividades de otros servicios ordinarios reside en: la necesidad de prestar un servicio universal; la obligación de garantizar la continuidad de la actividad; la necesidad de preservar la asequibilidad del servicio; y la especial protección de los derechos de los consumidores y de los usuarios respecto de dicho servicio.

- La identificación y definición de las misiones de servicio público susceptibles de ser protegidas en los SIEG recae sobre las autoridades nacionales. Sin embargo, las misiones específicas de servicio público no agotan el interés general presente en el sector, sino que, simplemente, se orientan a su máxima garantía en un mercado liberalizado.

- En el caso de los servicios de radiodifusión, la definición de misión de servicio público en las diferentes leyes audiovisuales es de carácter cualitativo, amplia y general. Sin embargo, dicha opción está prevista por la Comisión Europea por la especificidad del servicio en cuestión.

- Aunque como norma general la prestación de los SIEG debe satisfacerse dentro del habitual funcionamiento del mercado, se contempla la posibilidad de aplicar medidas excepcionales para garantizar la prestación de determinados servicios. En el caso de la televisión dichas medidas pueden incluir la definición y atribución de misiones de servicio público a un ente de titularidad estatal y su financiación extraordinaria.

- En lo referente a la financiación los Estados tienen libertad para optar por un sistema de financiación única (a cargo de los presupuesto del Estado) o por un sistema de doble financiación (que combine fondos públicos y comerciales). La Comisión Europea, encargada de vigilar la compatibilidad del sistema elegido con el Derecho comunitario, no cuestiona, a priori, ni uno ni otro.

- Las ayudas destinadas a satisfacer esas misiones de servicio público, para ser acordes con la normativa comunitaria, deben ajustarse a las exigencias de necesidad, transparencia y proporcionalidad. La Comisión Europea se atiene a estos criterios para evaluar si un sistema resulta compatible o no con el libre mercado.

Es decir, la opción de eliminar la publicidad de RTVE no constituye una necesidad que se derive de su configuración jurídica de acuerdo con el marco previsto para los SIEG, sino que resulta de una decisión de carácter político y empresarial que trae consigo otro tipo de problemas como puede ser la viabilidad económica del ente o la excesiva carga económica en la compensación que se le exige tanto a televisiones privadas como a operadores de telecomunicaciones con la finalidad de compensar la reducción de ingresos por la eliminación de los contenidos publicitarios. 


\section{Referencias: documentos y legislación}

- Carta de la Comisión Europea. AYUDA ESTATAL REINO DE ESPAÑA Ayuda Estatal C 38/09 (ex NN 58/09) Nuevo sistema de financiación basado en impuestos para los organismos de radiodifusión pública en España Invitación a presentar observaciones en aplicación del artículo 108, apartado 2, del TFUE (Texto pertinente a efectos del EEE) (2010/C 8/12). Consultada en: http://www.derecho.com/1/doue /ayuda-estatal-reino-espa\%D1a-ayuda-estatal-c-38-09-ex-nn-58-09-nuevo-sistema-financiacion-basado-impuestos-organismos-radiodifusion-publicaespa\%F1a-invitacion-presentar-observaciones-aplicacion-articulo-108-apartado-2tfue-texto-pertinente-efectos-eee-2010-c-8-12/

- COM (1996) 443 final, Comunicación de la Comisión sobre los servicios de interés general en Europa.

- COM (2000) 580 final, Modificación de la Comunicación de la Comisión sobre los servicios de interés general en Europa.

- COM (2001) 598 final. Informe de la Comisión al Consejo Europeo de Laeken sobre los servicios de interés general.

- COM (2003) 270 final. Libro Verde sobre los servicios de interés general.

- COM (2004) 374 final. Libro Blanco sobre los servicios de interés general.

- COM (2007)725 final. Un mercado único para la Europa del siglo veintiuno. Servicios de interés general, incluidos los sociales: un nuevo compromiso europeo.

- Comunicación de la Comisión Marco de la Unión Europea sobre ayudas estatales en forma de compensación por servicio público, DO C 8, de 11.01.2012, pp. 15-22.

- Comunicación de la Comisión relativa a la aplicación de las normas de la Unión Europea en materia de ayudas estatales a las compensaciones concedidas por la prestación de servicios de interés económico general, DO C 8. de 11.01.2012, pp. 4-14).

- Comunicación de la Comisión sobre la aplicación de las normas en materia de ayudas estatales a los servicios públicos de radiodifusión, DO C 257 de 27.10.2009, pp. 0001-0014.

- Comunicación de la Comisión sobre la aplicación de las normas en materia de ayudas estatales a los servicios públicos de radiodifusión, DO C 320 de 15.11.2001, pp. 0005- 0011.

- Decisión de la Comisión relativa a la aplicación de las disposiciones del artículo 106, apartado 2, del Tratado de Funcionamiento de la Unión Europea a las ayudas estatales en forma de compensación por servicio público concedidas a algunas empresas encargadas de la gestión de servicios de interés económico general, Do L 7 de 11.01.2012. pp. 3-10.

- Decisión 93/403/CEE, del 11 de junio de 1993, relativa a un procedimiento de aplicación del artículo 85.3 del Tratado, DO L 179, p. 23.

- Decisiones de la Comisión sobre ayudas estatales al servicio público de radiodifusión (1999-2010): http://ec.europa.eu/competition/sectors/media/decisions_psb.pdf 
- Directiva 2007/65/CE, del Parlamento Europeo y del Consejo, de 11 de diciembre de 2007, de Servicios de Medios Audiovisuales, DO L 332, de 18.12.2007, pp. 27-45.

- Directiva 2006/111/52/CE, relativa a la transparencia de las relaciones financieras entre los Estados Miembros y las empresas públicas, así como a la transparencia financiera de determinadas empresas, DO L 318 de 17.11.2006, pp.17-25.

- European Commission Competition Directorate-General: "Legal Framework conditions applicable to State aid to public service broadcasting", en: http://ec.europa.eu/competition/sectors/media/decisions_psb.pdf [Consulta: 3/03/2014].

- European Commission: "Competition in the Media Sector" en: http://ec.europa.eu/competition/sectors/media/overview_en.html [Consulta: 30/09/2013].

- Ley 7/2010, de 31 de marzo, General de Comunicación Audiovisual, BOE 1.04.2010, pp. 30157-30209.

- Ley 8/2009 de 28 de agosto de Financiación de la Corporación de Radio y Televisión Española, BOE 31.08.2009, pp. 74003-74015.

- Ley 17/2006, de 5 de junio, de la Radio y la Televisión de titularidad estatal, BOE 6.06.2006, pp. 21207-21227.

- Reglamento $n^{\circ} 360$ relativo a la aplicación de los artículo 107 y 108 del Tratado de Funcionamiento de la Unión Europea a las ayudas de minimis concedidas a la empresas que prestan servicios de interés económico general, DO L 114, de 26.04.2012, pp. 8- 13.

- Resolución del Parlamento Europeo sobre la Comunicación de la Comisión relativa a los servicios de interés general, A4-0357/97, DO C 014 de 19.01.1998, p. 0074.

- Versión consolidada del Tratado de Funcionamiento de la Unión Europea, DO C 115, de 9.5.2008, pp. 47-115.

\section{Referencias bibliográficas}

ARRIAZA IBARRA, Karen (2012): "Funding and governance of public service television in times of crisis: the cases of France, Spain, Germany and the United Kingdom". Media Transformations, vol. 8, pp. 4-22.

ECOTEUVE.es (16/09/2013): "RTVE prevé terminar el ejercicio 2013 con un déficit de al menos 70 millones", en: http://ecoteuve.eleconomista.es/ecoteuve/empresas/noticias/5145065/09/13/RTVE-preve-terminar-el-ejercicio-2013-con-un-def icit-de-al-menos-70-millones-.html\#.Kku8LWZjmG5215f [Consulta: 26 de marzo de 2014].

GÓMEZ BARROSO, José Luis \& MARBAN-FLORES, Raquel (2013): "Basic financial services: A new service of general economic interest?". Journal of European Social Policy, July, pp. 332-339.

MUÑOZ SALDAÑA, Mercedes (2011): "Key issues to understand the controversy surrounding the economic support of Spanish public television". Communication and Society/Comunicación y Sociedad, vol. XXIV, n. 1, 2011, pp.119-140. 